\title{
An Immune Algorithm Based on Entropy For Computing Offloading Problem in Edge Computing Scenes
}

\section{ZHU Sifeng}

Tianjin Chengjian University

CAl Jianghao ( 807079588@qq.com )

Tianjin Chengjian University https://orcid.org/0000-0001-7123-6211

SUN Enlin

Tianjin Chengjian University

ZHANG Qinghua

Tianjin Chengjian University

\section{Research Article}

Keywords: Mobile Edge Computing, Computing Offloading, Immune Algorithm(IA), Information Entropy, Workload balancing, Offloading delay

Posted Date: November 2nd, 2021

DOI: https://doi.org/10.21203/rs.3.rs-899987/v1

License: (c) (i) This work is licensed under a Creative Commons Attribution 4.0 International License. Read Full License 


\title{
An immune algorithm based on Entropy For Computing Offloading Problem in
}

\section{Edge Computing Scenes}

\author{
ZHU Si-feng ${ }^{1 a^{*}}$, CAI Jiang-hao ${ }^{1 \mathrm{a}}$, SUN En-lin ${ }^{\text {1a }}$, ZHANG Qing-hua ${ }^{\text {1b }}$ \\ (1a. School of Computer and Information Engineering, Tianjin Chengjian University, Tianjin, 300384, China \\ 1b. Library, Tianjin Chengjian University, Tianjin, 300384, China)
}

\begin{abstract}
By offloading computing tasks to the edge of mobile network, mobile edge computing(MEC) solves the shortcomings of mobile devices in computing capacity and storage capacity. In order to reduce the computing delay of mobile terminal devices and the degree of workload balancing in MEC servers, a computing offloading solution based on information entropy immune algorithm is proposed. Firstly, the system model, delay model, load model and objective optimization model are constructed. Then, a task offloading scheme based on immune optimization is proposed .Finally, this scheme is compared with the Benchmark scheme and the offloading scheme in the literature. Simulation experiments show that the proposed scheme can effectively reduce the average offloading delay, and can effectively balance the load between MEC servers. At the same time, compared with the schemes in the literature, the performance of the scheme is improved to a certain extent.
\end{abstract}

Keywords: Mobile Edge Computing, Computing Offloading, Immune Algorithm(IA), Information Entropy, Workload balancing, Offloading delay

\section{Introduction}

With the commercialization of $5 \mathrm{G}$ technology, a large number of mobile terminals with low delay and low energy consumption is appearing, and the computing capacity required by user terminals in all walks of life increases substantially. In order to cope with this demand, mobile edge computing was born. Mobile Edge Computing provides real-time and flexible computing for users through the deployment of lightweight cloud servers[1], At the same time, the computing resources and storage of the cloud service center are transferred from the center to the edge of the network[2].Shorten the data transmission distance and optimize the time required to complete the offloading service, which can better meet the needs of low latency applications. Edge computing has greater advantages than traditional cloud computing : (1) 
IOV devices will be processed in edge data centers or locally on the device, greatly reducing the pressure on the cloud. (2) Mobile edge computing will distribute data to different edge devices, so hackers will not be able to affect the entire service by attacking one device, which can better protect users' privacy. (3) Edge computing is used to transfer the pressure of the central server, relieve the pressure of network bandwidth, and expand the network. (4) Edge computing has better reliability. Edge data devices and Internet of Things devices are closer to users, which improves the response capability of the system and reduces the possibility of interruption, thus reducing the processing delay.

When multiple users access the mobile edge computing data center, a large number of computing tasks will be offloading on multiple servers of mobile edge computing, and a large number of concurrent computing load processing will be carried out. How to design such a computing mode is the problem encountered in mobile edge computing[3]. At present, the offloading decision-making problem about edge mobile computing scenario has had certain results: literature [4] on the system response time delay and energy consumption of mobile terminal comprehensive consideration, using genetic algorithms as optimization method, give full consideration to the workflow neutron task sequence and executive position, a system to minimize the response time delay is given and the task of mobile equipment energy consumption uninstall programs; In Literature [5], the problem of workload allocation and resource allocation in the heterogeneous network scenario of mobile edge computing integration was jointly discussed. Literature [6] adopts the adaptive offloading strategy based on genetic algorithm to process the effective traffic in the Internet of Things - infrastructure-cloud environment, so as to improve the success rate and reduce the algorithm complexity, delay and processing time. Literature [7] conducts a targeted study on the dependency relationship among various tasks, proposes a offloading strategy combining workflow scheduling, and uses genetic algorithm to optimize it. Literature [8] solves the problem of workload distribution in the fog cloud architecture of the Internet of Things. NSGAII algorithm is used as the optimization method to reduce the time delay and meet the energy consumption requirements under the three-layer architecture of the Internet of Things, fog and cloud. Literature [9] puts forward a three-tier architecture composed of vehicle cloud, roadside cloud and central cloud, and uses hybrid adaptive particle swarm optimization algorithm to complete resource allocation under this architecture, which can effectively reduce delay and ensure reasonable resource allocation. Literature [10] designed a vehicle-to-vehicle routing acquisition algorithm, which used the non-dominated sorting algorithm to effectively reduce the workload balancing rate and energy consumption of the server, and shorten the processing time of the computing task .Literature [11] proposed an AI-supported load 
optimization method, by performing spectrum allocation and optimal device-to-gateway configuration, the gateway detects the idle spectrum channel in the permitted frequency band to allocate it to IoT devices. Literature [12] proposes a new cross-task knowledge transfer mode based on denoising autoencoder for single-objective and multi-objective multi-task optimization. Literature [13] balances the relationship between energy consumption and time delay when the fog and cloud process the workload, and adopts NSGAII algorithm for effective processing.

The schemes proposed in the above literatures were considered from the aspects of system response delay, workload balancing of servers or mobile terminal energy consumption, etc., and the corresponding task offloading strategy was given. In this paper, the system response time delay and server workload balancing are considered comprehensively, and a task offloading scheme is proposed to minimize the system response time delay and workload balancing of servers.

Our main contributions are following:

- An edge computing offloading optimization model combining workload balancing and time delay is proposed.

- A computing offloading solution based on entropy immune algorithm optimization method is presented.

- An effective optimization algorithm evaluation scheme is proposed.

\section{Offloading decision model in Edge Computing Scenes}

In this section, the details of the offloading decision are divided into four parts for introduction. This paper proposes a multi-objective optimization model based on minimizing transmission delay and workload balancing or the problem of offloading decision in the MEC scenario.

\subsection{System model}

The system model designed in this paper is shown in Figure 1., assumed that the set of the user terminal devices is Users $=\left\{\right.$ user $_{1}$, user $_{2}, \ldots$, user $\left._{M}\right\}$ in the system , where user $_{m}(1 \leq m \leq M)$ is represented as an edge computing server.In one time period, the user has a computation-intensive task to offload from the server. Assuming a sufficient number of base stations are available, mobile users submit tasks to the nearest base station based on the strength of the signal. Technically, assumed that the set of edge computing servers is $M E C S=\left\{M E C_{1}, M E C_{2}, \ldots, M E C_{N}\right\}$, where $M E C_{n}(1 \leq n \leq N)$ is represented as an edge computing server. 
Each server has different storage and computing resources, and fiber optic links are used between servers to reduce delay. Has a collaborative server to coordinate the assignment of tasks within its jurisdiction. The collaborative server will record the available status of each MEC server, and reasonably allocate the tasks within its jurisdiction by considering the size of tasks submitted by users, the available space of each server, the workload of all servers and the main goal of reducing the delay. In addition, in order to simplify the analysis process and obtain valuable rules, this paper assumes that the process is quasi-static, that is, the user set remains unchanged in a time period, but the user set may change in different time periods.

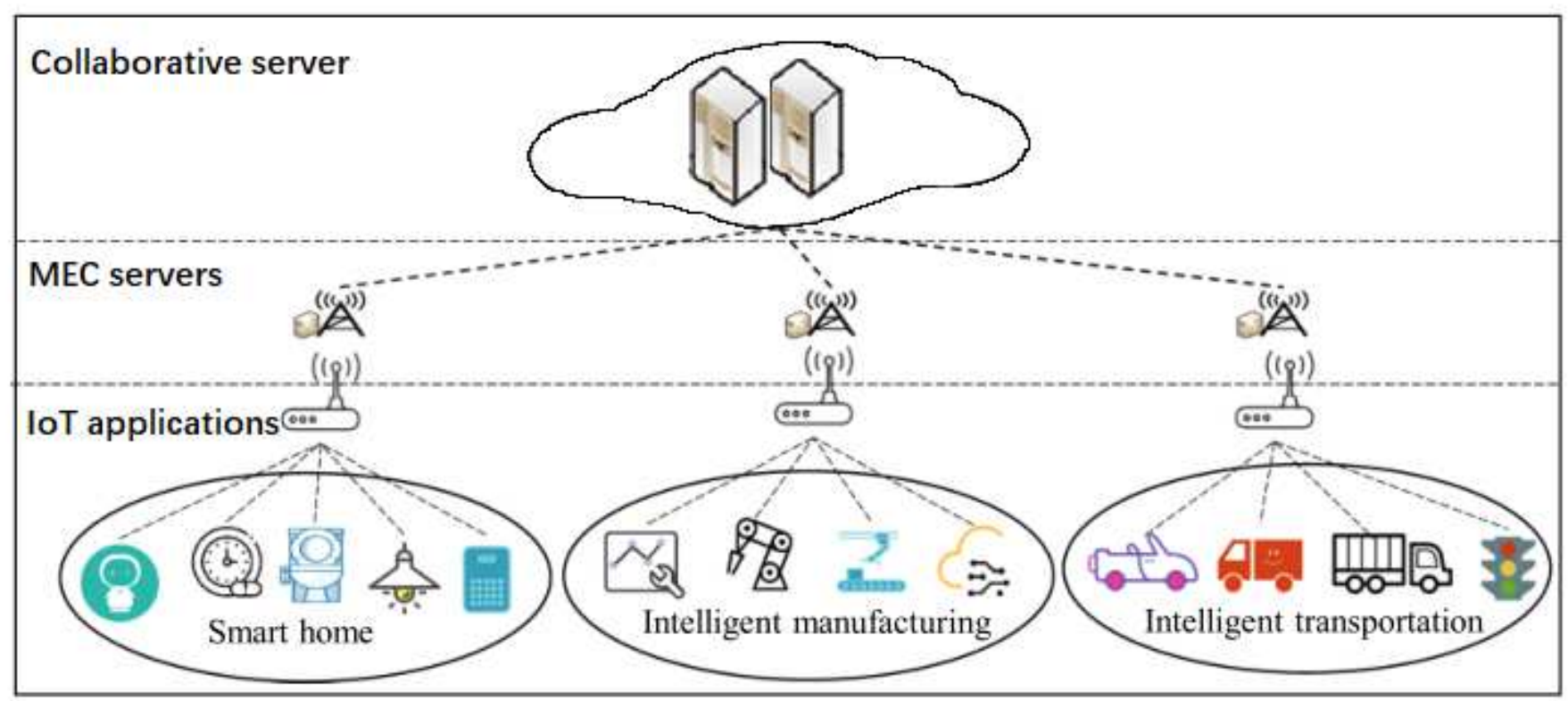

Fig. 1 System model

\subsection{Delay model}

Let the user $_{\mathrm{i}}$ have a computation-intensive task task $_{i}$ that needs to apply for offloading service from the edge server. This task needs storage resources $z_{\mathrm{i}}$ and computing resources $c_{\mathrm{i}}$, which are jointly expressed as $\operatorname{task}_{i}=<z_{i}, c_{i}>$. The MEC server has storage resources $Z_{M E C}^{j}$ and computing resources $C_{M E C}^{j}$ that can be offloaded by the user, which are jointly expressed as $M E C_{j}=<Z_{M E C}^{j}, C_{M E C}^{j}>, 1 \leq j \leq N$ 。 At the beginning of each period, the user submits his task to the nearest server, and the server submits the binary group $\left\langle z_{i}, c_{i}\right\rangle$ to the collaborative server, which determines the computing offloading strategy according to the remaining resources of each server and the user's resource requirements. Suppose k tasks to be completed are assigned to $M E C_{j}$, and the uncompleted tasks that have been 
offloaded are denoted as $\operatorname{Tasks}_{K}^{j}=\left\{\operatorname{task}_{1}^{j}, \operatorname{task}_{2}^{j}, \ldots, \operatorname{task}_{k}^{j}\right\}, \operatorname{tas}_{k}^{j}=<z_{k}^{j}, c_{k}^{j}>. \operatorname{task}_{i}$ can be offloaded to $M E C_{j}$ only when Equation (1) is satisfied.

$$
z_{i}+\sum_{k=1}^{K} z_{k}^{j}<Z_{M E C}^{j}, 1 \leq k \leq K, 1 \leq j \leq N
$$

In the Equation (1), $z_{k}^{j}$ is the storage resources occupied by $\operatorname{task}_{k}^{j}$ that has been allocated to $M E C_{j}$. The computing delay of user task is only related to the computing resources required and the processing performance of the server, so the computing delay $T P_{i}$ of $\operatorname{task}_{i}$ on $M E C_{j}$ is shown in Equation (2).

$$
T P_{i}=\frac{c_{i}}{C_{M E C_{j}}^{j}}
$$

Where $c_{i}$ is the computing resources required by $\operatorname{tas}_{i}$, and $C_{M E C}^{j}$ is the computing resources owned by $M E C_{j}$. According to Equation (2), queue delay $T Q_{i}$ of $\operatorname{task}_{i}$ on $M E C_{j}$ can be obtained as equation (3).

$$
T Q_{i}=\frac{1}{C_{M E C}^{j}} \sum_{k=1}^{K} c_{k}^{j}
$$

Set transmission delay is fixed as $T_{0}$, the execution delay $\tau_{i}$ of $\operatorname{tas}_{i}$ is shown in Equation (4).

$$
\tau_{i}=T_{0}+T P_{i}+T Q_{i}
$$

The total execution delay of all tasks can be expressed as Equation (5).

$$
T=\sum_{i=1}^{M} \tau_{i}
$$

\subsection{Load model}

In order to evaluate the workload degree of the model, it is divided into two parts: the use degree of the offloading scheme for all servers and the load task amount assigned to a single server. Let the vector group $Y=\left(y_{1}, y_{2}, \ldots, y_{j}, \ldots y_{N}\right)$, where the element $y_{j} \in\{0,1\}$, when $y_{j}=0$, means that no service is provided for any user in this offload, and when $y_{j}=1$, means that service is provided for at least one mobile user. Set the usage degree parameter $\chi_{\text {mec }}$ to represent the usage degree of all servers, as shown in Equation (6).

$$
\chi_{\text {mec }}=1-\frac{1}{N} \sum_{j=1}^{N} y_{j}
$$


The value of the parameter $\chi_{\text {mec }}$ will be 0 when all servers are in use and $\chi_{\text {mec }}$ will increase as the number of idle MEC servers increases. In other words, the lower the utilization rate of the number of servers is, the higher the parameter of usage degree $\chi_{\text {mec }}$ is and it tends to be 1.Let the vector group $A=\left(a_{1}, a_{2}, \ldots, a_{j}, \ldots a_{N}\right)$ represent the workload of all servers, as shown in Equation (7).

$$
a_{j}=\frac{1}{Z_{M E C}^{j}} \sum_{k=1}^{K} z_{k}^{j}, 1 \leq \mathrm{j} \leq N
$$

The vector group $A=\left(a_{1}, a_{2}, \ldots, a_{j}, \ldots a_{N}\right)$ is processed by the way of linear function normalization, as shown in Equation (8).

$$
\chi_{\text {load }}=\frac{1}{N}\left(\sum_{j=1}^{N} \frac{a_{j}-a_{\text {min }}}{a_{\max }-a_{\min }}\right)
$$

The workload balancing rate L of the server is shown in Equation (9).

$$
L=\frac{\chi_{\text {load }}}{1-\chi_{\text {mec }}}
$$

\subsection{Optimization model}

In this paper, the model designed in the moving edge computing scenario takes minimizing the delay and balancing the load situation of the server as two objectives to be optimized .In this model, the system response time delay and server load were comprehensively considered, and the weight coefficient was balanced to calculate the proportion between the time delay and load rate. After weighted processing, the two objectives were converted into a comprehensive cost objective, and a constrained optimization model was established to minimize the comprehensive cost, as shown in Equation (10).

$$
\left\{\begin{aligned}
& \min T, L \\
& \text { s.t. } \sum_{k=1}^{K} z_{k}^{j} \leq Z_{M E C}^{j}, 1 \leq k \leq K, 1 \leq j \leq N \\
& \sum_{k=1}^{K} c_{k}^{j} \leq C_{M E C}^{j}, 1 \leq k \leq K, 1 \leq j \leq N \\
& 0 \leq \omega_{1} \leq 1 \\
& 0 \leq \omega_{2} \leq 1 \\
& \omega_{1}+\omega_{2}=1
\end{aligned}\right.
$$

\section{Offloading decision scheme based on entropy immune algorithm}

In recent years, great progress has been made in the research of bionic intelligent optimization algorithms .Immune algorithm is one of them, it is inspired by the biological immune system, reference related concepts and form a new type of intelligent search algorithm, with adaptability, randomness, 
parallelism, global convergence and other characteristics, in the combinatorial optimization, structural design, artificial intelligence and other fields have been applied.

\subsection{Encoding}

Because the problem under study needs to consider both the delay and the workload balancing of each server .In the offloading decision process, each user-submitted task is assigned to each server and the information is offloaded to the collaborative server, and then their latency and workload balancing are calculated.The offload target for each task is represented by a group of vectors $X=\left(x_{1}, x_{2}, \ldots x_{M}\right)$, where $\mathrm{x}_{i}$ are decimal variables and $1 \leq x_{i} \leq N$.A vector group $Z=\left(z_{1}, z_{2}, \ldots, z_{M}\right)$ and $C=\left(c_{1}, c_{2}, \ldots, c_{M}\right)$ is added to the genome to complete the auxiliary parsing of the affinity function. The antibody $\mathrm{Ab}$ encoding is shown in Figure 2.

\begin{tabular}{|l|l|l|l|l|l|l|l|l|l|l|l|l|l|l|l|l|l|}
\hline$x_{1}$ & $x_{2}$ & $\cdots$ & $x_{\mathrm{i}}$ & $\cdots$ & $x_{M}$ & $z_{1}$ & $z_{2}$ & $\cdots$ & $z_{\mathrm{i}}$ & $\cdots$ & $z_{M}$ & $c_{1}$ & $c_{2}$ & $\cdots$ & $c_{\mathrm{i}}$ & $\cdots$ & $c_{M}$ \\
\hline
\end{tabular}

Fig. 2 Antibody code

\subsection{Affinity evaluation function}

In the immune algorithm, the affinity represents the binding strength of antibody and antigen, which corresponds to the fitness in the genetic algorithm. The affinity operator is usually expressed as a function .In this paper, the affinity evaluation function needs to be analyzed and designed from the two perspectives of minimizing the delay and maintaining the workload balance of each server, so the antibody affinity evaluation function Equation (11) is adopted.

$$
f(A b)=F(X, Y)=\omega_{1} F_{1}(X, Y)+\omega_{2} F_{2}(X, Y)
$$

\subsection{Mutation operator}

In this paper, the mutation operator is used to mutate the obtained antibody scheme, so as to increase the diversity of antibody group and realize local search. The variation probability $\mathrm{Pm}$ is expressed in Equation (12).

$$
P m=p m * \frac{g}{g_{\max }}
$$

In the Equation, $p m$ is the initial mutation probability, $g$ is the current iteration number, $g_{\max }$ and is the maximum iteration number. The design of the mutation operator will make the mutation probability decrease with the reduction of iteration number, so as to better realize local search. Decimal code is adopted for the offloading decision of antibody. The method for the variation operation of the offloading decision part $\mathrm{X}$ is to randomly assign a bit of antibody according to probability. The specific operation 
is shown in Figure 3.

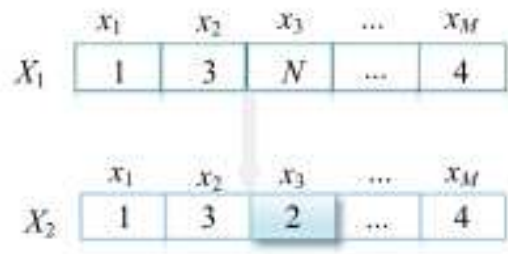

Fig. 3 Mutation

\subsection{Concentration evaluation operator}

The concentration of antibody can indicate the diversity of antibody population. When the concentration of a certain antibody is too high, it indicates that the antibody population tends to be over-concentrated, which may lead to local optimization and reduce the global search ability. Therefore, it is necessary to inhibit the high concentration of antibodies to ensure the diversity of antibody population. In this paper, the concept of information entropy is introduced to prevent the antibody concentration from being too high. Shannon proposed information entropy to measure and quantify information, and the measurement Equation of information entropy can be obtained as Equation (13)

$$
H(U)=-\sum \frac{1}{M} \log \frac{1}{M}
$$

Where, $\mathrm{M}$ is the number of mobile terminal devices. In this paper, it is necessary to reduce the total delay while keeping the workload of each server at similar level. Based on this basic goal, vector $\alpha=\left(k_{1}, k_{2}, \ldots, k_{j}, \ldots, k_{N}\right)$ is set, where $k_{j}$ represents the number of tasks assigned to $M E C_{j}$ in this scheme, and the evaluation operator of antibody concentration is expressed as Equation (14).

$$
\operatorname{den}(A b)=[\max (\alpha)-\min (\alpha)]^{*} H(U) * \exp (-g e n)
$$

\subsection{IA algorithm}

The basic flow of the IA algorithm is shown in the Fig. 4. 


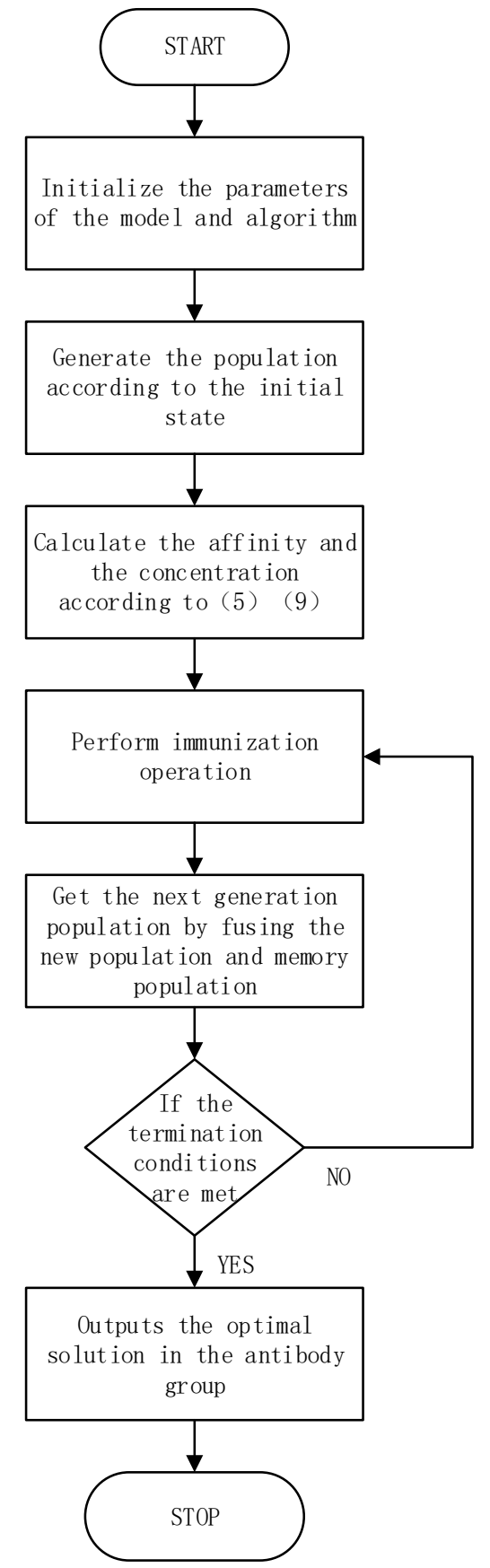

Fig. 4. The flow of the IA algorithm

\section{Performance evaluation}

In order to verify the effectiveness of the proposed improved immune genetic algorithm based on information entropy, that is, it can effectively reduce the offloading delay of mobile users and ensure the workload balancing of the server, this paper uses MATLAB to carry out simulation experiments on it. In this simulation experiment, the influence of different schemes on time delay and workload is firstly considered. Then, the impact of the number of servers on workload and delay was considered, namely, the number of servers was set as $N=10, N=20, N=40$. Secondly, considering the influence of weight coefficient on the experiment, the weights were set as $\omega 1=0.3, \omega 1=0.5$ and $\omega 1=0.7$. Finally, the 
role of information entropy in the improved design was verified. The system model is shown in Figure 1. The control condition is set to $N=20$ and $\omega 1=\omega 2=0.5$, while the number of mobile users is set to $M=100 \sim 1000$ (step size is 100). Each and cell is located in the same place, and mobile users are randomly distributed in each cell. Fiber optic links are used between each cell to ensure that task offloading delays are ignored. Detailed simulation parameters are shown in Table 1.

Table1 The simulation parameters

\begin{tabular}{|c|c|}
\hline Indicators & parameter \\
\hline Number of $M E C$ servers & $N=10,20,40$ \\
\hline Number of mobile users & $M=100,200,300,400,500,600,700,800,900,1000$ \\
\hline The mobile user requests computing resources $c_{i}$ & Random distribution between 1 and $6 /(M I P S)$ \\
\hline The mobile user requests a storage resource $\mathrm{Z}_{i}$ & Random distribution between 10 and $60 /(G B)$ \\
\hline MEC server computing resources $C_{M E C}^{j}$ & Random distribution between 100 and $600 /(M I P S)$ \\
\hline MEC server stores resources $Z_{M E C}^{j}$ & Random distribution between 1000 and $6000 /(G B)$ \\
\hline The objective function $F_{1}(X, Y)$ assigns weights & $\omega 1=0.3,0.5,0.7$ \\
\hline The objective function $F_{2}(X, Y)$ assign weights & $\omega 2=0.7,0.5,0.3$ \\
\hline
\end{tabular}

In this section, the scheme based on information entropy immune algorithm (IA-entropy) is compared with the benchmark (Benchmark) scheme and the scheme based on mixed integer linear programming ( MILP) in literature [5]. The following describes the offloading scheme used:

- Benchmark: All tasks are offloaded to the nearest server. However, if overload is found during uninstallation, it will be uninstalled to an adjacent server. This process is repeated until all tasks have been assigned.

- Mixed Integer Linear Programming (MILP) :In literature [5], an offloading scheme is proposed for this model and the intlinProg () function in MATLAB is used to deal with this $0-1$ integer linear programming problem more efficiently.

In this section, the weighted sum of task delay and server load is taken as the comprehensive cost of task execution, and the comprehensive cost of (IA-entropy) scheme, (MILP) scheme and（Benchmark） scheme are compared under the same conditions. The following is a comparative experiment from the changes in task number, server number and weight coefficient. 


\subsection{The influence of different schemes on delay and workload balancing}

As the number of mobile users continues to increase, the performance of the improved IA algorithm and benchmark scheme also changes, and the required delay is shown in Figure 5. When the number and weight of servers are kept the same, the average offloading delay required increases with the increase of the number of users, the offloading service pressure is constantly increasing, and the workload balancing is constantly rising. However, the delay reduction caused by the improved IA offloading algorithm is constantly increasing. When the number of users reaches 1000 , The average offloading delay of the improved scheme is $30.98 \%$ higher than that of the benchmark scheme, and the performance is $10.96 \%$ higher than that of the MILP-based scheme in Literature [5].

The performance comparison of workload balancing of MEC server is shown in Figure 6. Offloading algorithms tend to allocate tasks to high-performance servers while balancing tasks, which increases the workload imbalance rate. However, as the number of tasks continues to increase, the queuing delay of high-performance servers continues to rise, and some tasks are offloaded to idle servers, and the rise of the curve slows down. Second, the improved algorithm tends to select more servers and allocate tasks proportionally to all servers. However, with the increasing number of users, the workload balancing of the improved offloading algorithm is always lower than that of the benchmark scheme and MILP scheme

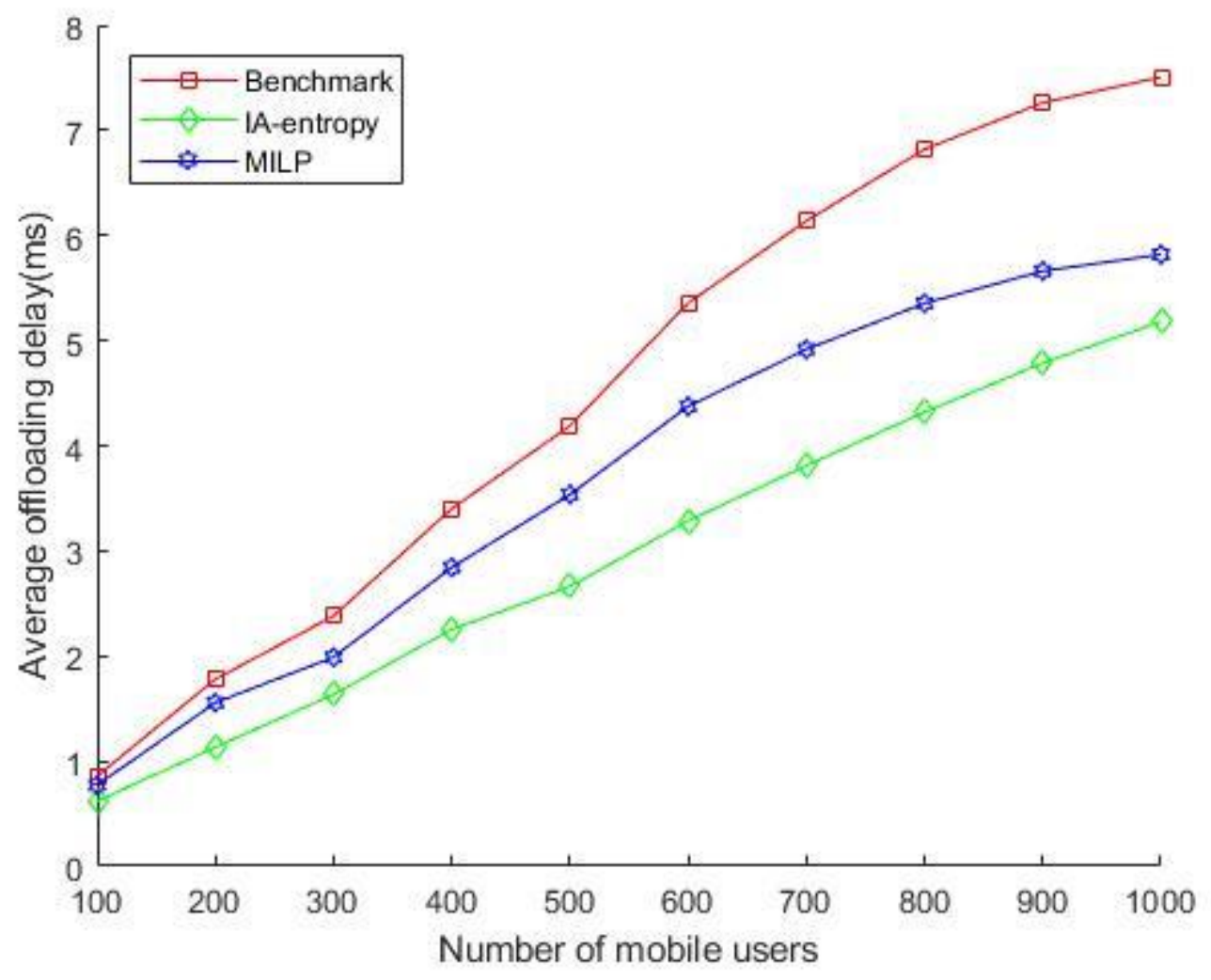

Fig. 5 Comparison of Average offloading delay between different schemes $(\mathrm{N}=20)$ 


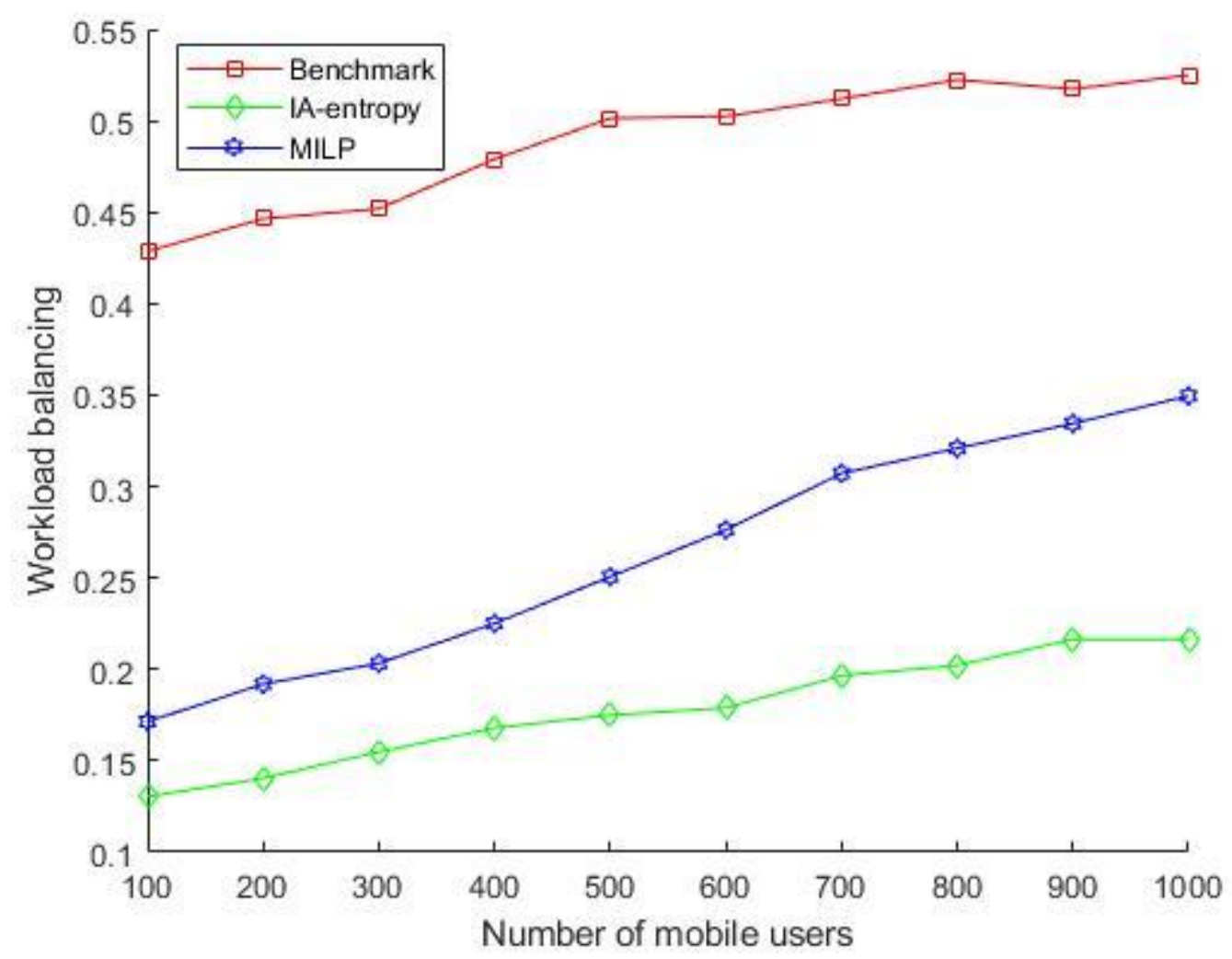

Fig.6 Comparison of workload balancing between different schemes $(\mathrm{N}=20)$

\subsection{Influence of different weights on time delay and workload balancing}

In order to analyze the influence of weight changes on the experiment, $\omega_{1}=0.3$ and $\omega_{1}=0.7$ were respectively set in the following experiment, and $\omega_{1}=0.5$ was added as the control, and the other conditions remained unchanged to explore the influence of different weights on the average offloading delay and workload balancing on the experimental band.

As shown in Figure 7, when the number of mobile users and the number of MEC servers are the same, if the weight of delay is increased, that is, increasing $\omega_{1}$ will reduce the average offload delay. When the number of mobile users keeps increasing, this phenomenon tends to be obvious. When the number of mobile users reaches 1000 , the average offloading delay of $\omega_{1}=0.7$ increases by $3.09 \%$ compared with that of $\omega_{1}=0.5$. On the contrary, reducing the weight $\omega_{1}$ will increase the average offloading delay. When the number of mobile users is 1000 , the average offloading delay of $\omega_{1}=0.3$ decreases by $3.33 \%$ compared with that of $\omega_{1}=0.5$.

Figure 8 shows the influence of weight on workload balancing rate. When the number of mobile users and servers is the same, increasing weight $\omega_{2}$ will enable the algorithm to allocate tasks in a 
more balanced way. When the number of mobile users is the same, the workload balancing of $\omega_{2}=0.7$ is always smaller than that of $\omega_{2}=0.5$ or $\omega_{2}=0.3$. If the weight of $\omega_{2}$ is reduced, the workload balancing of the server decreases and the average offloading delay increases.

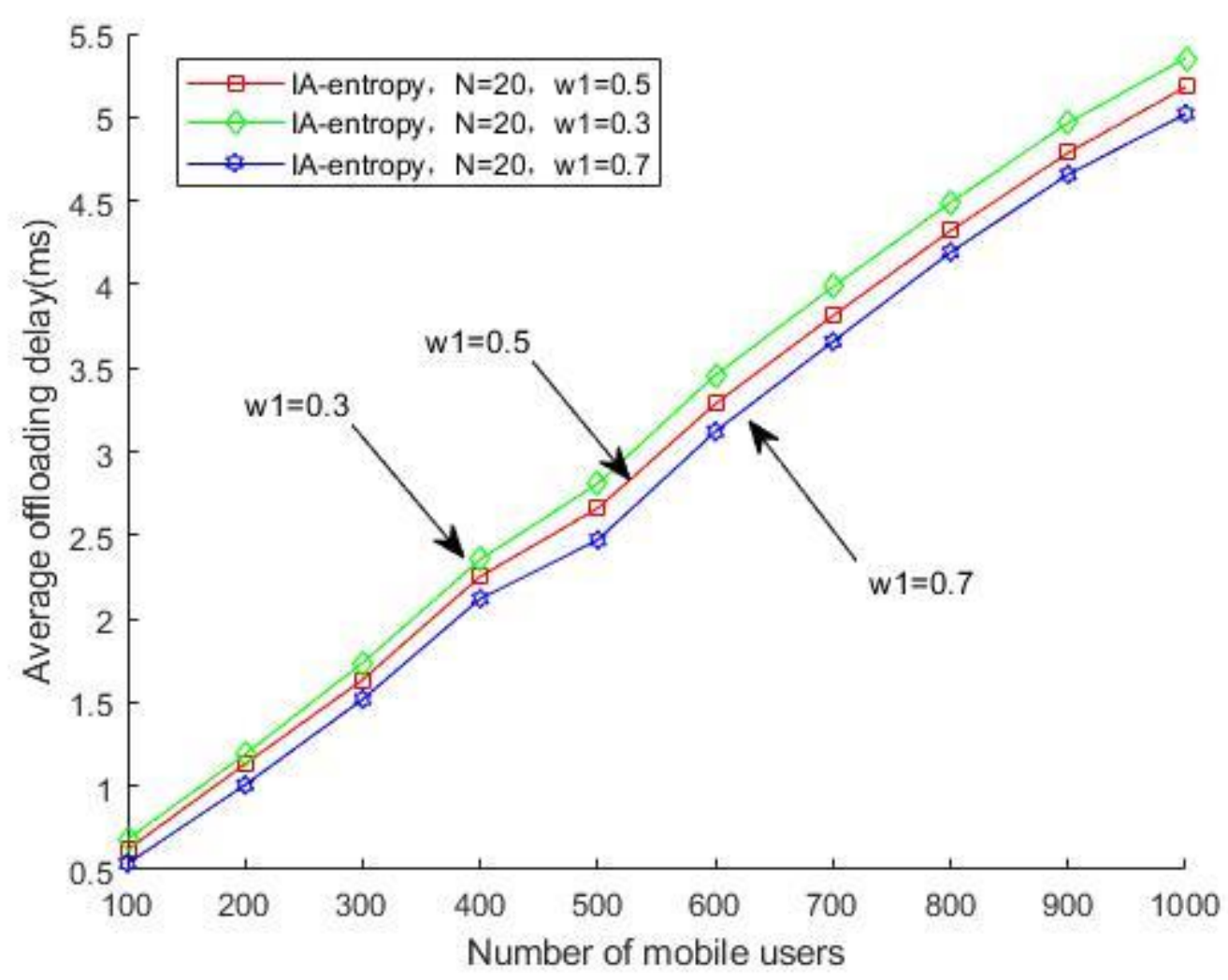

Fig.7 Comparison of average offloading delay with different weights $(\mathrm{N}=20)$ 


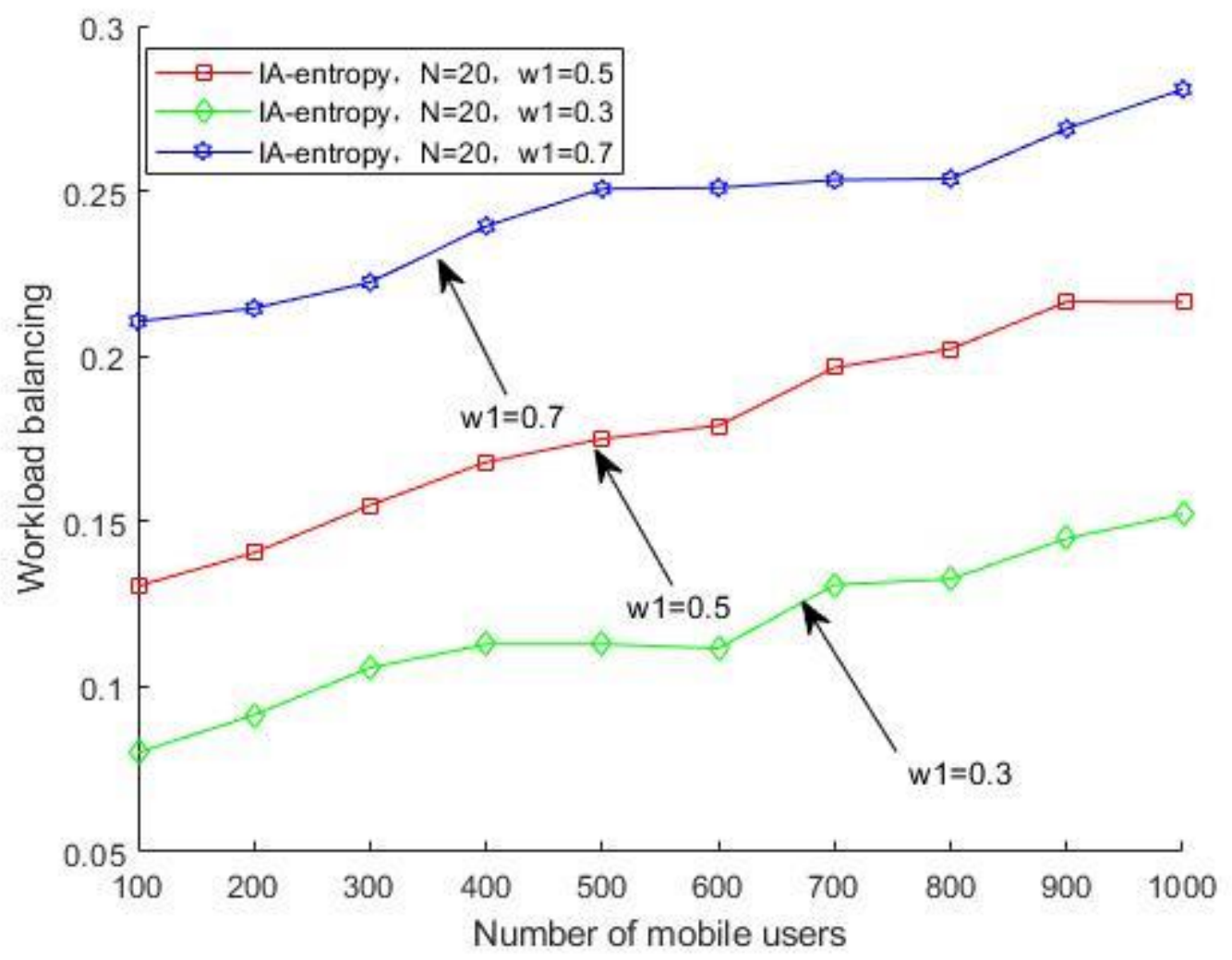

Fig. 8 Comparison of workload balancing with different weights $(\mathrm{N}=20)$

\subsection{The impact of the number of servers on delay and workload balancing}

Figure. 9 explores the influence of the number of servers on the existence of delay. As the number of mobile users continues to increase, the pressure of offloading services continues to increase, and the average offloading delay also increases with the increase of the number of users. When the number of users increases from 100 to 1000 , the average uninstallation delay of 40 servers increases from $44.86 \%$ to $47.04 \%$ compared with the average uninstallation delay of 20 servers, and the average uninstallation delay of 20 servers increases from $17.71 \%$ to $44.81 \%$ compared with the average uninstallation delay of 10 servers. The impact of the number of MEC servers on the average offloading delay tends to be significant, indicating that the increase of the number of servers will significantly reduce the processing delay of tasks.

Figure 10 shows that the number of servers has a significant impact on the degree of server workload balancing. With the increase of the number of tasks, the workload balancing of the server shows a certain degree of improvement, indicating that the more the number of tasks to be processed by each server, the more the solution tends to allocate tasks to high-performance servers, resulting in a certain workload unbalancing. As the number of servers increases, the workload balancing of servers decreases effectively, indicating that the increase in the number of servers can effectively reduce the 
workload of each server.

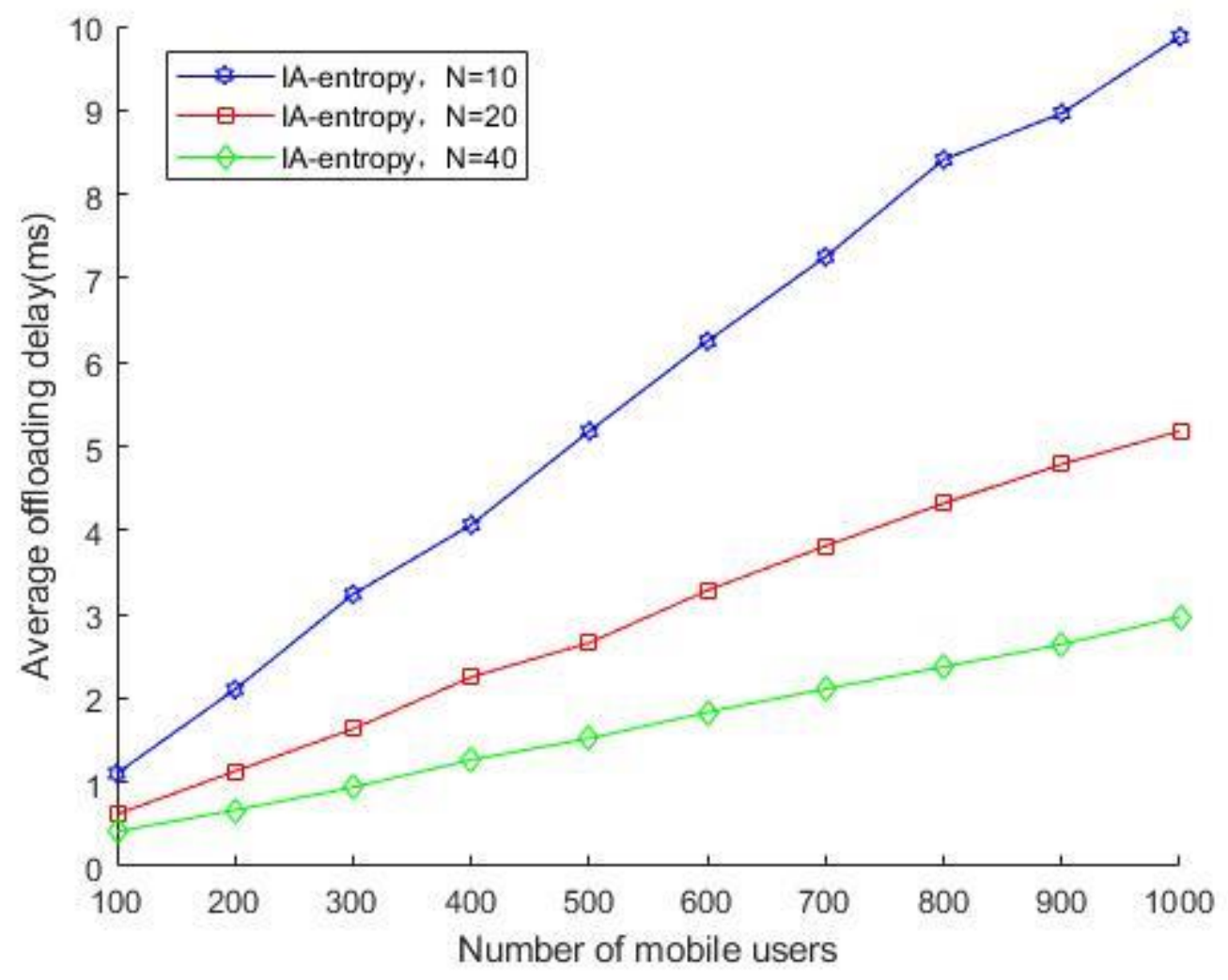

Fig.9 Comparison of average offloading delay with different server numbers

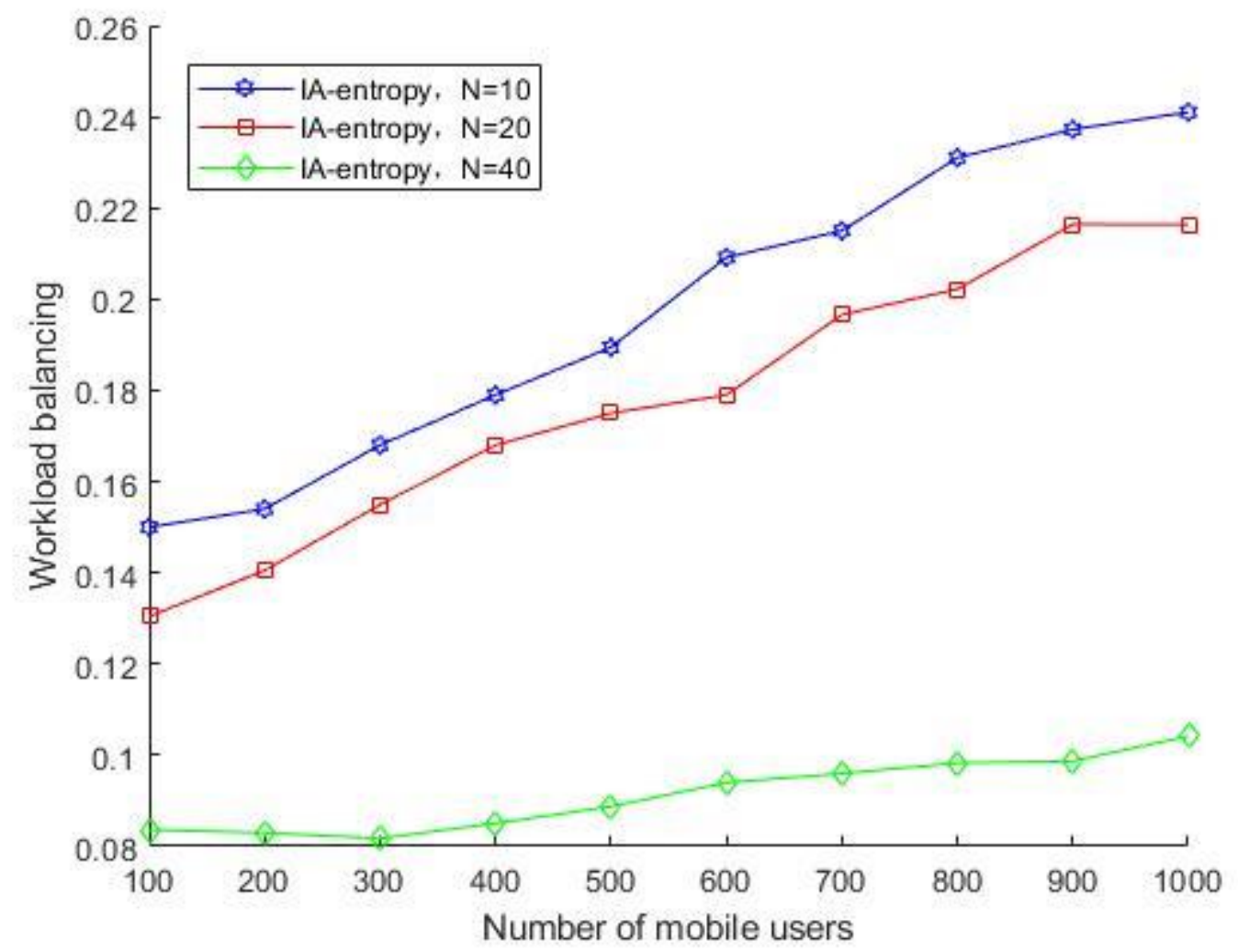

Fig.10 Comparison of workload balancing with different server numbers 


\subsection{The influence of entropy improvement on immune algorithm}

In order to verify the influence of information entropy factor on immune algorithm, this section conducts 100 independent comparison experiments between the scheme based on information entropy immune algorithm and the scheme based on immune algorithm. The evaluation indicators adopted are described below:

- Dominated in Number of Effective Generation (DEG): The optimal value of the immune algorithm does not change every iteration in the iterative process. The effective iteration refers to whether the optimal affinity of the antibody group breaks through. The set of effective iterations is denoted as $N E G=\left\{n e g_{1}, n e g_{2}, \ldots, n e g_{g}\right\}, 1<g<g_{\max }$, Where $n e g_{g}$ is binary variable, indicating whether a new optimal value is generated in this iteration. The dominant number of effective iterations mentioned in this section refers to whether the number of effective iterations of the immune algorithm based on information entropy is greater than that of the immune algorithm scheme in the 100 iterations of a single experiment, which is expressed as Equation (15).

$$
D E G=\left\{\begin{array}{l}
1, \text { if } \sum N E G_{\text {IA-entropy }}>\sum N E G_{\mathrm{IA}} \\
0, \text { otherwise }
\end{array}\right.
$$

- Dominated in Number of Better Generation (DBG) : The number of excellent iterations refers to the number of times that the immune algorithm scheme based on information entropy is superior to the immune algorithm scheme based on immune algorithm in 100 iterations of a single experiment. The set of excellent iterations is denoted as $N E G=\left\{n b g_{1}, n b g_{2}, \ldots, n b g_{g}\right\}$, $1<g<g_{\max }$ where $n b g_{g}$ is a binary variable to indicate whether scheme IA-entropy is superior to scheme IA in this iteration, and $\mathrm{x}$ is the interval to determine whether scheme IA-entropy is superior in a single experiment. DBG is expressed as Equation (16).

$$
D B G=\left\{\begin{array}{l}
1, \text { if } \sum N E G \in x \\
0, \text { otherwise }
\end{array}\right.
$$

In this section, the same initial values are used in the experiment. Each time, various parameters are set as , $M=400$ and $\omega_{1}=\omega_{2}=0.5$ comparative iteration. The number of iteration ranges from 20 to 100 (step size is 20), and the data is correlated. The experimental data are shown in Table 2. 
Table2 Experimental data

\begin{tabular}{ccccc}
\hline iteration & DEG & DBG, $0 \leq \mathrm{x} \leq 5$ & DBG, $6 \leq \mathrm{x} \leq 15$ & $\mathrm{DBG}, 16 \leq \mathrm{x} \leq 20$ \\
$0-20$ & 54 & 24 & 59 & 17 \\
$20-40$ & 57 & 31 & 41 & 28 \\
$40-60$ & 57 & 29 & 36 & 35 \\
$60-80$ & 66 & 33 & 33 & 34 \\
$80-100$ & 49 & 32 & 29 & 39 \\
\hline
\end{tabular}

Table 2 shows The Times of excellence of 100 independent experiments in various evaluation indicators in every 20 iterations. DEG of scheme based on the information entropy immune algorithm is better than that of scheme based on the immune algorithm. In the interval of 80-100 iterations, due to the decreasing weight of the information entropy, scheme based on the immune algorithm has the same local search ability at the later stage of the experiment. The analysis of DEG in different iteration intervals shows that the immune algorithm with the information entropy factor has better initial value adjustment ability, which can make the scheme get faster and better results.

The data of DBG presents certain symmetry. For example, in the interval of 0-20 iterations, the excellent iteration number of scheme based on information entropy in the interval of $0 \leq x \leq 5$ is the excellent iteration number of scheme based on immune algorithm in the interval of $16 \leq x \leq 20$. The data showed that at the beginning of the experiment, the addition of information entropy factor will cause DBG of the immune algorithm scheme based on information entropy is inferior to that of the immune algorithm scheme. and the end of the experimental information entropy factors impact on the algorithm of exponential decline, immune algorithm based on information entropy solution performance started rising, and the dominant frequency have risen sharply. The conclusion shows that the information entropy factor can dynamically condition the initial state and make the immune algorithm have better global search ability. Figure 11 shows the relationship between the number of iterations and system performance of an illustrative experiment. It can be seen from the figure that the algorithm has some disadvantages in the early stage, but after a certain number of iterations, scheme based on the information entropy immune algorithm obtains better objective function value. 


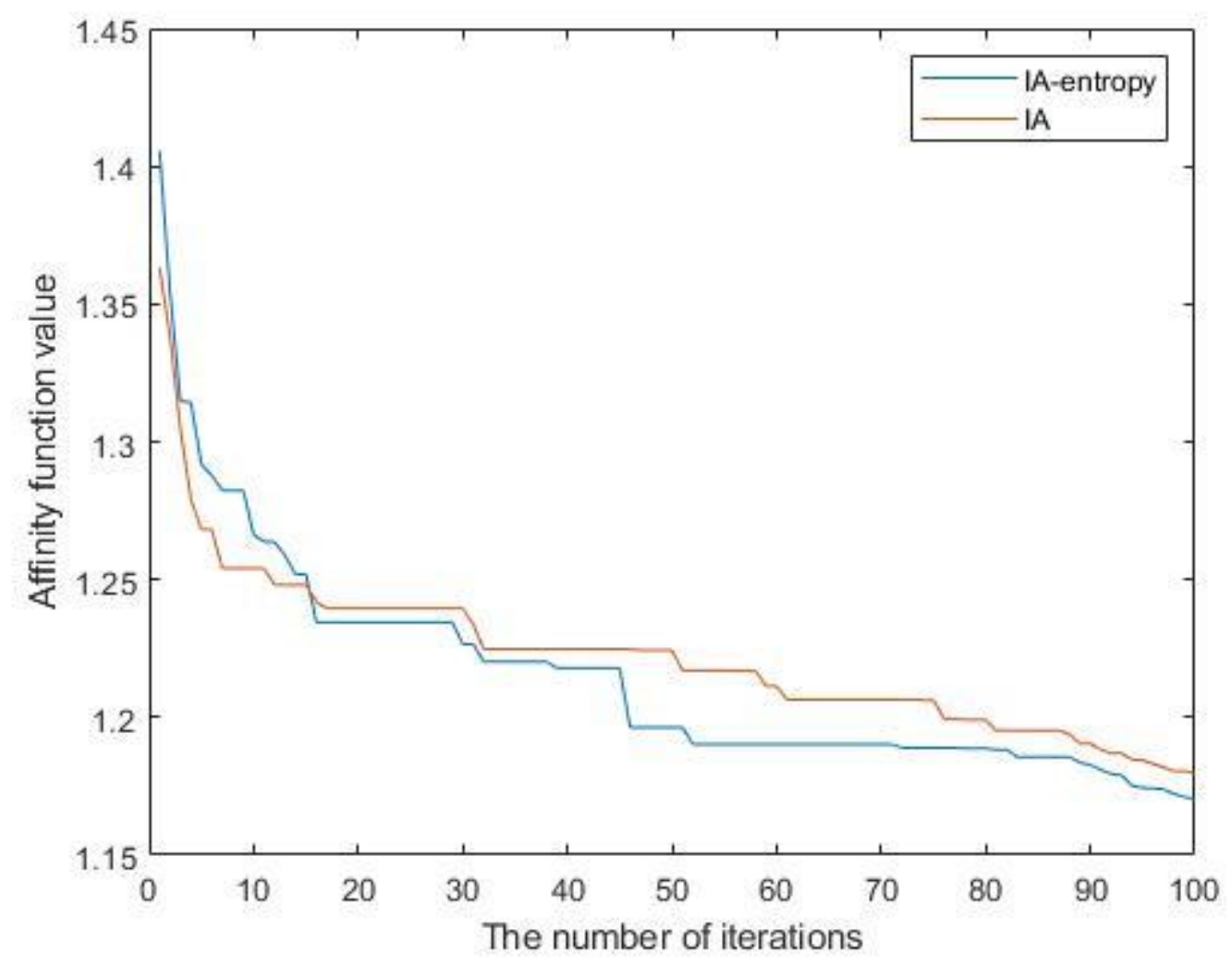

Fig.11 Comparison of iteration and system performance

\section{Conclusion}

In this paper, the parallel offloading problem of multiple mobile users is discussed in the mobile edge computing scenario, and the MEC system model is designed. Based on this model, the general goal is to minimize the mobile user delay and maintain the workload balancing of the server. The multiobjective optimization function is constructed to calculate the offloading method, and then the linear weighting method is used to transform it into a single-objective linear optimization problem, and the information entropy based immune algorithm is used to calculate the optimal solution. The simulation results show that the computational offloading scheme designed in this paper can effectively reduce the offloading delay, make reasonable use of all servers and keep the workload balancing rate of each server in a reasonable range. Compared with the benchmark scheme and the offloading scheme in literature [5], the offloading scheme based on the information entropy immune algorithm proposed in this paper has better performance and better application value. In the next step, the multi-objective optimization method will be used to solve the multi-user offloading problem in the scenario of moving edge calculation. 


\section{Acknowledgement}

This work was supported by the National Natural Science Foundation of China (grant numbers 61972456).

\section{Declaration of interests}

The authors declare that they have no known competing financial interests or personal relationships that could have appeared to influence the work reported in this paper.

The authors declare have no financial interests/personal relationships which may be considered as potential competing interests.

\section{Author biographies}

ZHU Si-feng earned his Ph.D. degree from school of computer science, Xidian university, Xi' an China, in 2012. Now, he is a professor in Tianjin Chengjian University, Tianjin, China. His research interests include mobile edge computing, internet of things and multi-objective optimization algorithm. He has published more than 20 papers in some international journals. E-mail: zhusifeng@tcu.edu.cn.

CAI Jiang-hao received his B.S. degree from Zhonghuan Information College Tianjin University of Technology, in 2020. Currently, he is pursuing the M.S. degree in software engineering at School of Computer Science and technology, Tianjin Chengjian University, Tianjin, China. His research interests include mobile edge computing and multi-objective optimization algorithm. E-mail: 807079588@qq.com.

SUN En-lin received his B.S. degree from Xingtai University, in 2019. Currently, he is pursuing the M.S. degree in software engineering at School of Computer Science and technology, Tianjin Chengiian University, Tianjin, China. His research interests include mobile edge computing and multi-objective optimization algorithm. E-mail: 1138896661@qq.com.

ZHANG Qing-hua received her B.S. degree from Zhoukou normal University, Henan, China. Now, she works at Tianjin Chengjian University, Tianjin, China. Her research interests include library management informatization, artificial intelligence algorithm. E-mail: 304854105@qq.com.

\section{References}

[1] ABBAS N, ZHANG Y, TAHERKORDI A, et al. Mobile Edge Computing: a Survey[J]. IEEE Internet of Things Journal, 2018,5(1):450-465.

[2]. Engineering - Electrical Engineering; Studies from Chongqing University of Posts and Telecommunications Provide New Data on Electrical Engineering (An Actor-critic Deep Reinforcement Learning Approach for Transmission Scheduling In Cognitive Internet of Things Systems)[J]. News of Science, 2020.

[3] ALTHUMALI H, OTHMAN M. A Survey of Random Access Control Techniques for Machine-toMachine Communications in LTE/LTE-A Networks[J]. IEEE Access, 2018,6:74961-74983.

[4] ZHOU Y, LI Z, GE J, et al. Multi-Objective Workflow Scheduling Based on Delay Transmission in Mobile Cloud Computing[J]. Ruan Jian Xue Bao/Journal of Software, 2018, 29(11): 3306-3325.

[5] ZHANG Wenzhu, CAO Beibei, YU Jianhua. Multi-user computation offloading approach for mobile edge com-puting[J].JOURNAL OF XIDIAN UNIVERSITY, 2020, 47(6): 1-7.

[6] Azham Hussain, et al."Genetic algorithm based adaptive offloading for improving IoT device communication efficiency." Wireless Networks .prepublish(2019):. doi:10.1007/s11276-019-02121-4. 
[7] DONG Hao, ZHANG Haiping, LI Zhongjin, et al. Computation offloading for service workflow in mobile edge computing[J]. Computer Engineering and Applications, 2019, 55(2): 36-43.

[8] ]Mahdi Abbasi,Ehsan Mohammadi Pasand,Mohammad R. Khosravi. Workload Allocation in IoTFog-Cloud Architecture Using a Multi-Objective Genetic Algorithm[J]. Journal of Grid Computing,2020(prepublish).

[9] Sadip Midya,Asmita Roy,Koushik Majumder,Santanu Phadikar. Multi-objective optimization technique for resource allocation and task scheduling in vehicular cloud architecture: A hybrid adaptive nature inspired approach[J]. Journal of Network and Computer Applications,2018,103.

[10]Xiaolong Xu,Renhao Gu,Fei Dai,Lianyong Qi,Shaohua Wan. Multi-objective computation offloading for Internet of Vehicles in cloud-edge computing[J]. Wireless Networks: The Journal of Mobile Communication, Computation and Information,2020,26(5).

[11]Wei Yao,Fazlullah Khan,Mian Ahmad Jan,Nadir Shah,Izaz ur Rahman,Abid Yahya,Ateeq ur Rehman. Artificial intelligence-based load optimization in cognitive Internet of Things[J]. Neural Computing and Applications,2020,32(20).

[12]Feng Liang,Zhou Lei,Zhong Jinghui,Gupta Abhishek,Ong Yew-Soon,Tan Kay-Chen,Qin A K. Evolutionary Multitasking via Explicit Autoencoding.[J]. IEEE transactions on cybernetics,2018.

[13]Mahdi Abbasi,Ehsan Mohammadi Pasand,Mohammad R. Khosravi. Workload Allocation in IoTFog-Cloud Architecture Using a Multi-Objective Genetic Algorithm[J]. Journal of Grid Computing,2020(prepublish).

[14]Mahdi Abbasi,Ehsan Mohammadi Pasand,Mohammad R. Khosravi. Workload Allocation in IoTFog-Cloud Architecture Using a Multi-Objective Genetic Algorithm[J]. Journal of Grid Computing,2020(prepublish).

[15]Wei Yao,Fazlullah Khan,Mian Ahmad Jan,Nadir Shah,Izaz ur Rahman,Abid Yahya,Ateeq ur Rehman. Artificial intelligence-based load optimization in cognitive Internet of Things[J]. Neural Computing and Applica-tions,2020,32(20).

[16]Feng Liang,Zhou Lei,Zhong Jinghui,Gupta Abhishek,Ong Yew-Soon,Tan Kay-Chen,Qin A K. Evolutionary Multi-tasking via Explicit Autoencoding.[J]. IEEE transactions on cybernetics,2018.

[17]Zohreh Masoumi,Carlos A. Coello Coello,Ali Mansourian. Dynamic urban land-use change management using multi-objective evolutionary algorithms[J]. Soft Computing,2020,24(6).

[18]Fotis Foukalas. Cognitive IoT platform for fog computing industrial applications[J]. Computers and Electrical Engineering,2020,87.

[19]LIU L, ZHOU Y, YUAN J, et al. Economically Optimal MS association for Multimedia Content Delivery in Cache-Enabled Heterogeneous Cloud Radio Access Networks[J], IEEE JSAC,2019, 37(7): 1584-1593. 